\title{
VULNERABILIDADE SOCIOAMBIENTAL NA BACIA DO RIO JAPARATUBA EM SERGIPE A PARTIR DA ÁLGEBRA DE MAPAS
}

\author{
Christiane Freitas Pinheiro de Jesus ${ }^{1}$, Nelson Wellausen Dias², Marcus Aurelio Soares Cruz ${ }^{3}$
}

\section{RESUMO}

A bacia do rio Japaratuba teve grande parte de sua área desmatada dando lugar às pastagens, plantação de canade-açúcar e exploração mineral (petróleo e potássio). Com o intuito de realizar uma avaliação que contemplasse não somente os aspectos biofísicos, mas também fatores socioeconômicos, este estudo teve como objetivo realizar uma análise preliminar voltada para a avaliação da vulnerabilidade socioambiental dessa bacia. Para tanto, foram utilizadas técnicas de análise espacial que envolveram a álgebra de mapas sobre planos de informação que incluíam: a distribuição espacial da população e das pessoas sem renda por setor censitário, o uso da terra e o modelo de perda de solo por erosão. Os resultados obtidos demonstraram que os maiores valores de vulnerabilidade estiveram concentrados a partir do médio curso da bacia até alguns trechos nas proximidades da foz. Nessas áreas ocorre maior ocupação populacional e um maior número de pessoas sem rendimentos mensais fixos, além de maiores valores de perda de solo por erosão.

Palavras-chave: Análise integrada, bacia hidrográfica, geoprocessamento

\section{ABSTRACT \\ ENVIRONMENTAL VULNERABILITY IN THE JAPARATUBA RIVER BASIN OF SERGIPE BASED ON THE ALGEBRA OF MAPS}

Much of the Japaratuba river basin had been deforested in the past to make way space for pasture, sugarcane plantation and mineral exploration (oil and potassium). In order to perform an evaluation encompassing not only the biophysical aspects, but also the socioeconomic factors, this study sought to perform a preliminary analysis focused on the socioenvironmental vulnerability of this basin. Therefore, spatial analysis techniques involving map algebra of spatial information layers were conducted that included: spatial distribution of the population and people with no income per census sector, land use and soil loss by erosion model. The results showed that the vulnerability highest were concentrated in the central portion of the basin and towards some stretches near the mouth. In these areas there is higher population concentration, greater number of people without fixed monthly income, and higher values of soil loss by erosion.

Keywords: Integrated analysis, watershed, GIS

\section{Recebido para publicação em 23/05/2013. Aprovado em 18/12/2013.}

1 - Geógrafa, Analista em Geoprocessamento, IBGE, Aracaju - SE, christiane.jesus@ibge.gov.br

2 - Oceanógrafo, Analista em Geoprocessamento, IBGE, Aracaju - SE, nelson.dias@ibge.gov.br

3 - Engenheiro. Civil, Pesquisador, Embrapa, Aracaju - SE, marcus.cruz@embrapa.br

50 REVENG

ENGENHARIA NA AGRICULTURA, VIÇOSA - MG, V.22 N.1, JANEIRO / FEVEREIRO 2014 


\section{INTRODUÇÃO}

Uma das principais atividades agrícolas que ocorre tradicionalmente na bacia hidrográfica do rio Japaratuba é a criação de gado bovino e de cultivo da cana-de-açúcar desde o século passado (FONTES, 1997). A permanência dessas atividades é decorrente, principalmente, das condições ambientais favoráveis ao desenvolvimento de solos férteis e à disponibilidade de água. Aragão e Almeida (2009) destacam que a modificação da cobertura natural do solo pela exploração mineral de petróleo e potássio tem favorecido o aumento de volume do escoamento superficial e o consequente aumento na carga de sedimentos nos rios causando assoreamento, o que contribui para o aumento da vulnerabilidade do ambiente físico. Modificações desfavoráveis da cobertura natural da superfície e aplicação de técnicas inapropriadas de uso do solo, como aração morro abaixo, favorecem os processos erosivos e consequente perda de solo (HOLLY, 1980).

O termo vulnerabilidade é geralmente definido como uma situação em que estão presentes três elementos (ou componentes): exposição ao risco, incapacidade de reação e dificuldade de adaptação diante da materialização do risco (MOSER, 1998). A vulnerabilidade socioambiental integra as dimensões: social e ambiental, portanto, requer a utilização de metodologias de integração na análise.

A aplicação de técnicas de geotecnologia para a análise integrada de dados espaciais amplia nossa capacidade de analisar não somente grandes volumes de dados, mas também dados associados a fenômenos complexos. O desenvolvimento dessa tecnologia tem permitido construir modelos cada vez mais sofisticados que procuram reproduzir os fenômenos do mundo real usando dados com localização geográfica conhecida (georreferenciados) e com elevado grau de detalhamento nos atributos. Diversas aplicações tem sido desenvolvidas para a análise de fenômenos demográficos e sociais, como a análise de segregação espacial (FEITOSA et al., 2007; REARDON; O'SULLIVAN, 2004; WONG, 2005) e modelos espaço-temporais baseados em especificações algébricas (FERREIRA et al., 2013; HORNSBY; COLE, 2007).

A utilização da álgebra de mapas permite a análise conjunta e integrada de fenômenos espaciais, utilizando um conjunto de operadores que manipulam campos geográficos e tem sido amplamente adotada para estudos de vulnerabilidade. Halim et al. (2007), estimaram o risco de erosão do solo na bacia do rio Kaligarang, na Indonésia, por meio da análise integrada de fatores biofísicos e socioeconomicos. Seus resultados indicaram que os fatores população e renda foram os mais determinantes, dentre os parâmetros socioeconômicos, na avaliação dos riscos de erosão do solo.

No Brasil, são vários os estudos que fazem uso da álgebra de mapas, especialmente na definição de fragilidade ambiental e/ou com foco em zoneamentos. Pode-se citar, como alguns exemplos, o trabalho de Amaral et al. (2012) no qual foram utilizadas imagens de sensoriamento remoto e dados censitários para aprimorar a representação da distribuição populacional na Amazônia; de Gonçalves et al. (2011) para a determinação da fragilidade ambiental em bacias hidrográficas por meio da álgebra de mapas; de Becker e Egler (1997) voltados para definição de metodologia adotada pela Secretaria de Assuntos Estratégicos da Presidência da República - SAE, no zoneamento ecológico econômico da Amazônia Legal; de Barbosa et al. (1996) utilizando operadores zonais com álgebras de mapas também para fins de zoneamento ecológico econômico.

No entanto, a integração de dados físicos com dados socioeconômicos na escala de setores censitários, por meio da álgebra espacial, ainda tem se mostrado incipiente. Portanto, este trabalho tem como objetivo avaliar, preliminarmente, a vulnerabilidade socioambiental na bacia hidrográfica do rio Japaratuba, utilizando dados socioeconômicos do Censo 2010 e aplicando álgebra de mapas entre os seguintes temas: distribuição espacial da população e das pessoas sem renda por setor censitário, uso da terra e risco de perda de solo por erosão.

\section{MATERIAL E MÉTODOS}

A bacia do rio Japaratuba, localiza-se na região nordeste do estado de Sergipe (Figura 1), possui área de $1.674,2 \mathrm{~km}^{2}$ e drena 18 municípios (Figura 2) de pequeno e médio portes. Tal área corresponde por 768 setores censitários (Figura 3). De acordo com Aragão e Almeida (2009), 9,63 \% da bacia estão localizados na região semi-árida, 30,18 \% no litoral úmido e $60,17 \%$ no agreste, sendo que a precipitação total anual varia de 400 a $1.400 \mathrm{~mm}$. Seus principais afluentes são os rios Japaratuba (Alto e Baixo curso), Siriri e Japaratuba-Mirim, que 
constituem, também, as unidades de planejamento definidas pela SEMARH (2011).

Para a determinação da vulnerabilidade socioambiental da bacia hidrográfica, foram utilizados os seguintes materiais: dados de população e renda por setor censitário (IBGE, 2011); classificação do uso da terra, a partir de imagens SPOT, realizada pela SEMARH (Secretaria de Estado do Meio Ambiente e dos Recursos Hídricos) e publicada no Atlas Digital Sobre Recursos Hídricos de Sergipe (SEMARH, 2011); e a estimativa de perda de solo na bacia calculada pela EUPS (Equação Universal de Perda de Solo), como produto do projeto intitulado "Aplicação de ferramentas de geoprocessamento e de modelagem matemática na avaliação dos impactos ambientais decorrentes das atividades antrópicas na bacia do rio Japaratuba em Sergipe,
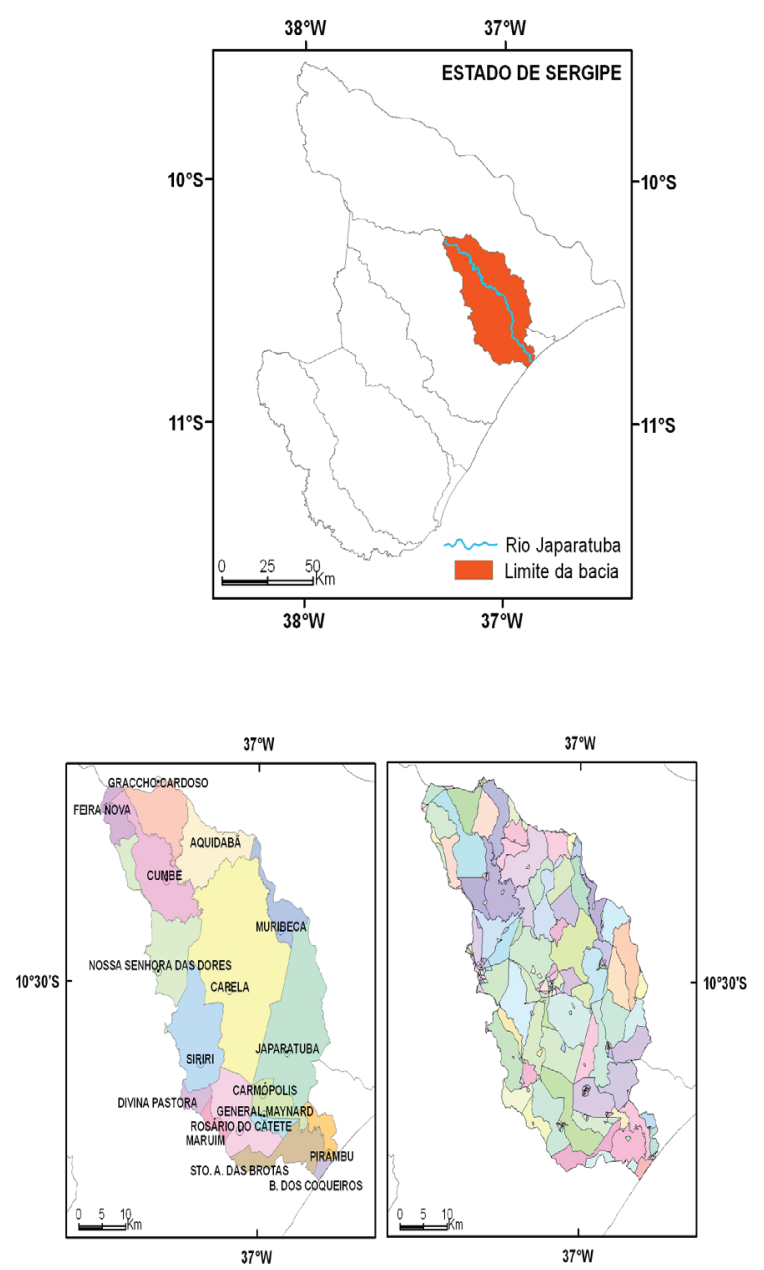

Figura 2. Divisão municipal

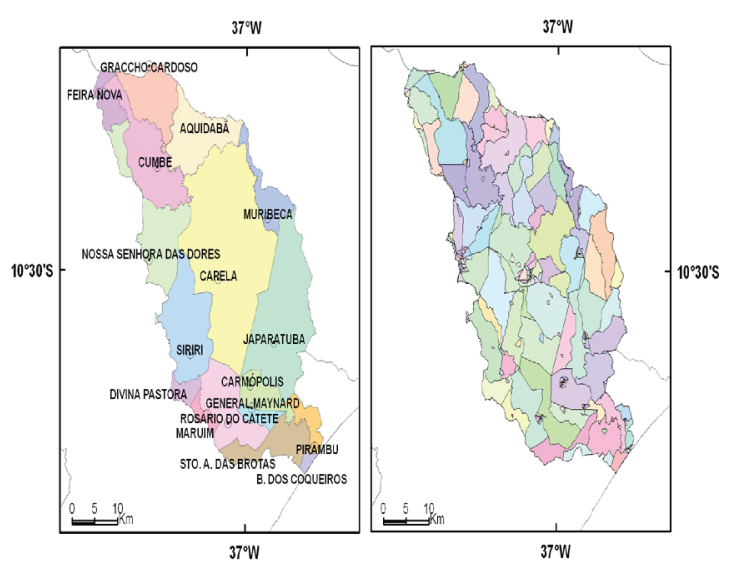

Figura 3. Divisão dos setores censitários

Considerando que diversos setores censitários podem estar inseridos parcialmente dentro de uma mesma bacia, foi necessário ponderar os dados de população e renda a partir do número de Unidades Visitadas (UV) por setor. Para tanto, foi dividido o número de UV que efetivamente estava dentro da bacia pelo total de UV do respectivo setor. Esta operação resultou num valor em percentual que, por sua vez, foi aplicado aos valores absolutos de população e renda para cada setor censitário localizado parcialmente na bacia do Japaratuba.

A partir daí, pôde-se dar início ao diagnóstico da vulnerabilidade socioambiental, que partiu da análise integrada dos temas: população, renda, EUPS e uso da terra utilizando a álgebra de mapas. A preparação dos dados passou pelas seguintes etapas:

(1) tratamento dos dados de população e renda - com os valores obtidos a partir da ponderação, associados a centróides criados para cada polígono dos setores. Foi feita a interpolação dos centróides pelo método IDW (Inverse Distance Weight Inverso Ponderado pela Distância). Este método de interpolação leva em consideração a dependência espacial dos valores interpolados por meio da determinação de pesos $(p)$ atribuídos aos pontos amostrados de forma proporcional à contribuição de cada valor vizinho, em função do respectivo valor e da distância, assim a distância recebe como expoente um valor '- $p$ ' que determina o grau de ponderação, de maneira que a soma dos pesos vizinhos seja igual a um (MENEGOL, 2004). Esta interpolação foi realizada pela ferramenta IDW, disponível no software ArcGIS versão 10, por meio da extensão Spatial Analyst. O uso do IDW neste estudo seguiu as orientações de Jacob e Young (2006), que destacam a necessidade de eliminar o denominado "efeito mosaico" ou "efeito xadrez", presente, em 
geral, na visualização de mapas temáticos, além de enfatizar as principais concentrações espaciais de população e renda, suavizando suas diferenças;

(2) Generalização das classes do uso da terra - as 17 classes de uso da terra identificadas pela SEMARH (2011) foram agrupadas em duas novas classes: Áreas Antropizadas (associação de caatinga/cultivos/pastagem, cultivos agrícolas/ solos expostos, pastagem, povoado/distritos, sede municipal, viveiro/salina, área degradada e área industrial) e Áreas Naturais (caatinga arbustiva arbórea, corpos d'água, dunas e areial, floresta estacional, floresta ombrófila, manguezal, mata ciliar, vegetação de restinga e área embrejada). O agrupamento das classes objetivou condensar a análise em dois cenários principais: um de maior alteração antrópica (Áreas Antropizadas) e outro de menor alteração (Áreas Naturais);

(3) Classificação temática dos valores da EUPS - os valores de perda do solo por erosão determinados pelo projeto da Embrapa Tabuleiros Costerios variaram de 0 a $3.965 \mathrm{t} \mathrm{ha}^{-1} \mathrm{ano}^{-1} \mathrm{e}$ foram agrupados em cinco categorias temáticas com base no histograma de distribuição de todos os valores subdivido em quintis, a saber: Perda Baixa (0 26,25 t ha ${ }^{-1}$ ano $^{-1}$ ), Perda Moderadamente Baixa (26,26 - 107,75 tha ${ }^{-1}$ ano $\left.^{-1}\right)$, Perda Mediana (107,76 - 282,72 $\mathrm{t} \mathrm{ha}^{-1}$ ano $\left.^{-1}\right)$, Perda Moderadamente Alta $\left(282,73-666,36 \mathrm{t} \mathrm{ha}^{-1}\right.$ ano $\left.^{-1}\right)$ e Perda Alta $(666,36$ $-3.965 \mathrm{t} \mathrm{ha}^{-1} \mathrm{ano}^{-1}$ ).

Todas as classes identificadas em cada tema foram consideradas como campos geográficos na álgebra de mapas e foram constituídas por um conjunto de pixels que agregam os respectivos valores de sua classificação temática, já que foram transformados de formato vetorial para formato matricial ou raster. Para realizar a álgebra de mapas dos temas, fez-se uso da ferramenta Raster Calculator, disponível na função Map Algebra acionada por meio da extensão Spatial Analyst do ArcGis 10. Posteriormente, foi realizada uma reclassificação dos valores, os quais foram reagrupados em três classes $(0,1$ e 2$)$ utilizando-se, para isso, a ferramenta Reclassify. $\mathrm{Na}$ Tabela 1 mostram-se os valores agrupados para cada classe dos respectivos temas e sua valoração segundo a contribuição à maior ou menor Vulnerabilidade Socioambiental (VSA), conforme critérios adotados neste estudo preliminar.

Quadro 1. Escala de valores para execução da álgebra de mapas

\begin{tabular}{|c|c|c|c|}
\hline TEMA & INTERVALOS & $\begin{array}{c}\text { VALOR DA } \\
\text { CLASSE }\end{array}$ & $\begin{array}{c}\text { DENOMINAÇÃO DA CLASSE } \\
\text { DE CONTRIBUIÇÃO À } \\
\text { VULNERABILIDADE } \\
\text { SOCIOAMBIENTAL }\end{array}$ \\
\hline \multirow{5}{*}{$\begin{array}{c}\text { POPULAÇÃO } \\
\text { (Número total de } \\
\text { pessoas) }\end{array}$} & $0,01-274,07$ & 0 & Pequena \\
\hline & $274,08-416,81$ & 0 & Moderadamente pequena \\
\hline & $416,82-570,97$ & 1 & Mediana \\
\hline & $570,98-747,97$ & 2 & Moderadamente grande \\
\hline & $747,98-1.455,97$ & 2 & Grande \\
\hline \multirow{5}{*}{$\begin{array}{c}\text { RENDA } \\
\text { (Número de } \\
\text { pessoas sem renda) }\end{array}$} & $0,01-104,83$ & 0 & Pequena \\
\hline & $104,84-159,43$ & 0 & Moderadamente pequena \\
\hline & $159,44-220,59$ & 1 & Mediana \\
\hline & $220,60-292,66$ & 2 & Moderadamente grande \\
\hline & $292,67-556,92$ & 2 & Grande \\
\hline \multirow{2}{*}{ USO DA TERRA } & Áreas naturais & 0 & Pequena \\
\hline & Áreas antropizadas & 1 & Mediana \\
\hline \multirow{5}{*}{$\begin{array}{c}\text { EUPS } \\
\left(\mathrm{t} \mathrm{ha}^{-1} \mathrm{ano}^{-1}\right)\end{array}$} & $0-26,25$ & 0 & Pequena \\
\hline & $26,26-107,75$ & 0 & Moderadamente pequena \\
\hline & $107,76-282,72$ & 1 & Mediana \\
\hline & $282,73-666,36$ & 2 & Moderadamente grande \\
\hline & $666,36-3.965$ & 2 & Grande \\
\hline
\end{tabular}


Após a reclassificação dos valores a operação algébrica foi realizada conforme a equação 1 :

$\mathrm{VSA}=($ pop_reclass + pess_s_r_reclass + uso reclass + USLE_reclass)

em que,

VSA = vulnerabilidade socioambiental; pop_reclass $=$ contribuição da população; pess_s_r_reclass $=$ contribuição das pessoas sem renda;

uso reclass $=$ contribuição do uso da terra; e

USLE_reclass $=$ contribuição da erosão do solo estimada pela EUPS.

Assim, os procedimentos metodológicos aplicados até chegar-se ao modelo de vulnerabilidade socioambiental, podem ser sintetizados no fluxograma representado na Figura 4:

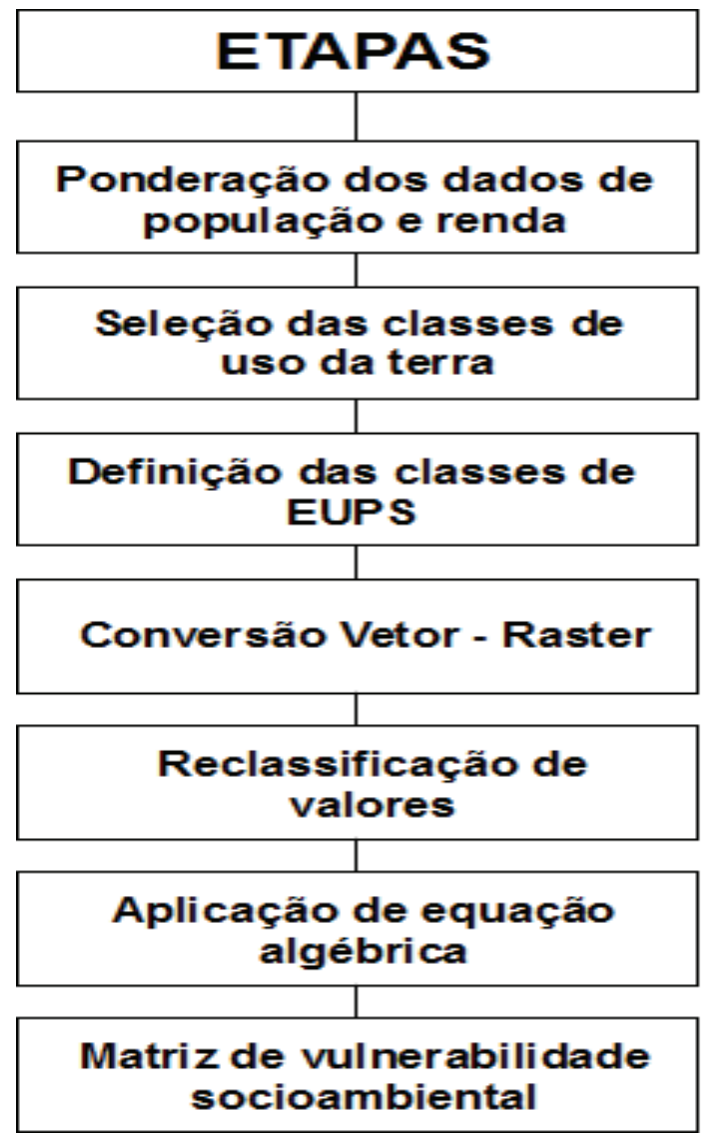

Figura 4. Síntese dos procedimentos metodológicos

\section{RESULTADOS E DISCUSSÃO}

O resultado da equação foi uma matriz com os valores variando de 0 a 7 , sendo que, quanto mais próximos de zero menor a contribuição dos fatores à vulnerabilidade socioambiental e quanto mais próximo de sete, maior esta contribuição. Estes valores foram reagrupados em 5 classes temáticas com base na distribuição de frequência. Dessa forma, as duas classes com menor frequência (classes 0 e 3 ) foram agrupadas com as classes Baixa (0 a 1) e Moderada (2 a 4), respectivamente. As classes resultantes retratam a qualificação dada aos graus de vulnerabilidade socioambiental, conforme representado na Figura 5. Portanto, as classes de menor grau de vulnerabilidade socioambiental são as que apresentam os menores valores dos fatores contribuintes, ou seja, menor população, menor proporção de pessoas sem renda, cobertura do solo mais natural e menor volume de perda de solo por erosão. Inversamente, as classes com maior grau de vulnerabilidade socioambiental apresentam valores maiores e cobertura do solo mais antropizada. A participação relativa de cada classe na área total da bacia é apresentada no Quadro 2.

Os resultados mostraram que $28,12 \%$ da área total da bacia foi classificada com VSA Moderadamente Alta. Esta categoria está associada às áreas de maior concentração populacional e onde existe um número maior de pessoas sem qualquer rendimento fixo mensal. Estas são áreas mais antropizadas que coincidem, principalmente, com as sedes urbanas de alguns municípios e seus respectivos entornos - Carmópolis, Pirambu, General Maynard, Japaratuba, Rosário do Catete, Siriri, Capela, Nossa Senhora das Dores, Muribeca e Cumbe - e com áreas de cultivos agrícolas associados a solos expostos. Porções significativas dessas áreas estão associadas à exploração de petróleo, no município de Carmópolis, e a de potássio, no município de Rosário do Catete.

Parte da borda sudeste da bacia e alguns trechos pequenos no município de Capela, onde a EUPS apresentou os maiores valores de perda de solos por erosão, foram classificadas como áreas com Alta VSA. Esses trechos, porém, restringem-se a cerca de $0,17 \%$ da área total da bacia.

A VSA decresce gradativamente à medida que se afasta destes núcleos de concentração, principalmente à montante, na parte setentrional 


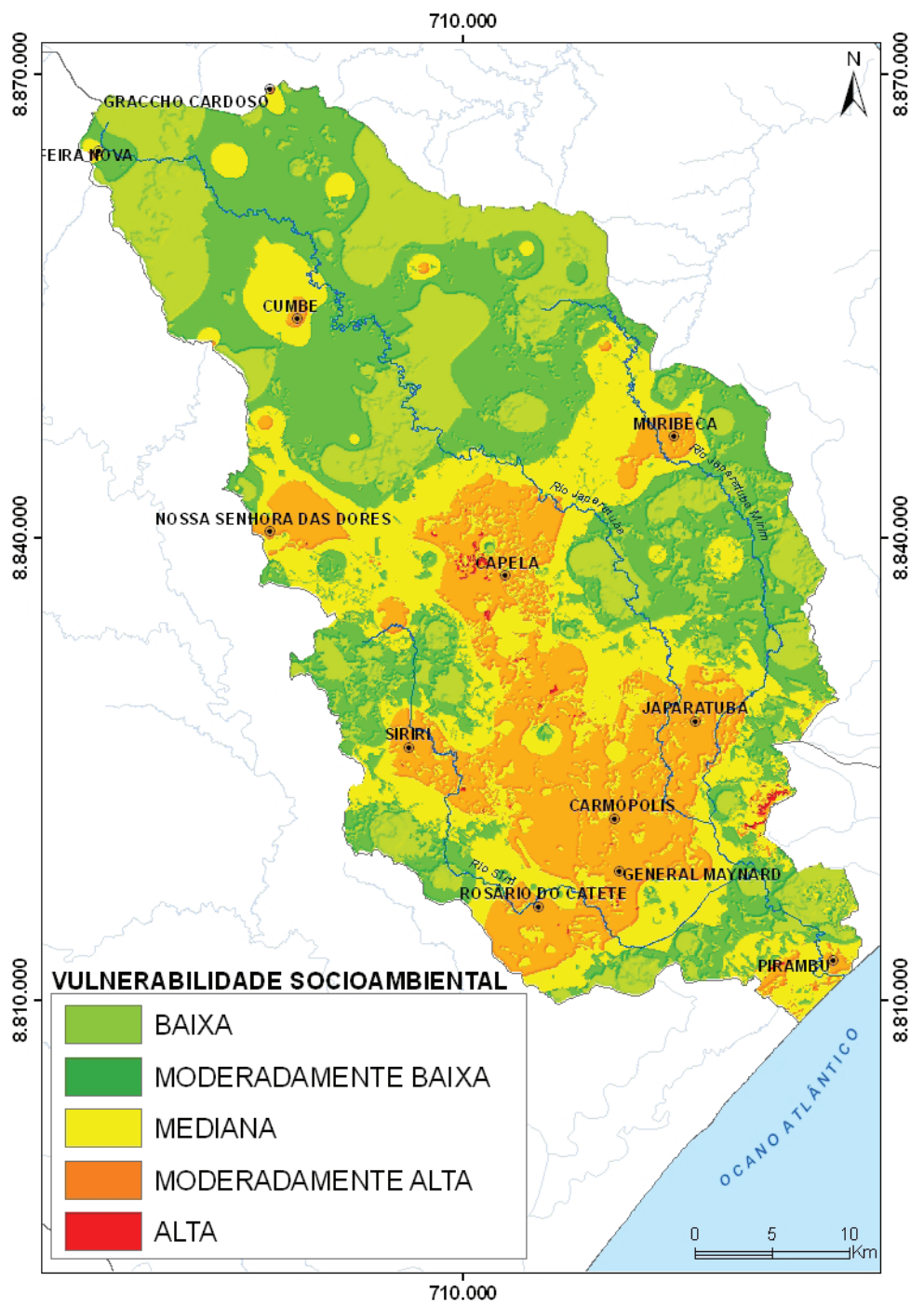

Figura 5. Vulnerabilidade Socioambiental (VSA) na bacia do rio Japaratuba-SE

Quadro 2. Quantificação do resultado por percentual em área da bacia hidrográfica do rio Japaratuba-SE

\begin{tabular}{ccc}
\hline AREA $\left(\mathbf{k m}^{\mathbf{2}}\right)$ & Área $\mathbf{~ e m ~} \%$ & Vulnerabilidade \\
\hline 394,24 & 23,71 & BAIXA \\
524 & 31,51 & MODERADAMENTE BAIXA \\
274,28 & 16,49 & MEDIANA \\
467,58 & 28,12 & MODERADAMENTE ALTA \\
2,9 & 0,17 & ALTA \\
\hline
\end{tabular}

da bacia. A partir daí, as classes variaram de Baixa $(23,71 \%$ de área $)$ a Moderadamente Baixa (31,51\%) e Mediana (16,49\%). Nessas regiões, apesar de serem registradas as maiores elevações (em torno de $250 \mathrm{~m}$ ), prevalecem os setores censitários tipicamente rurais com menor população e pessoas sem renda. O uso da terra está relacionado essencialmente à agropecuária, sendo que são encontradas áreas ainda naturais, com floresta estacional e caatinga arbustiva arbórea, o que favorece a cobertura do solo, diminuindo perdas por erosão. 
Assim, considerando as variáveis analisadas, percebe-se que as áreas mais vulneráveis concentram-se na região do médio curso da bacia e alguns trechos próximos à foz do rio. Este fato pode ser explicado, por serem regiões de maior ocupação populacional sem renda fixa mensal e por coincidir com alguns trechos onde a perda de solo por erosão apresentou-se elevada, comparada com o restante da bacia. Esses resultados indicam que possivelmente a ocupação de uma área por pessoas sem renda fixa poderia ser um fator agravante da vulnerabilidade de uma bacia hidrográfica.

\section{CONCLUSÕES}

- A álgebra de mapas demonstrou ser uma ferramenta eficaz na análise integrada de variáveis ambientais e socioeconômicas selecionadas para avaliar a vulnerabilidade socioambiental na bacia do rio Japaratuba;

- A execução dos procedimentos metodológicos apresentou um quadro de vulnerabilidade que varia atualmente de baixa à alta, sendo que, a maior parte dela encontra-se no estágio de VSA moderadamente baixa $(31,51 \%)$, seguida pela classe moderadamente alta $(28,12 \%)$;

- Por essa avaliação preliminar pode-se afirmar que a bacia não apresenta grandes impactos decorrentes da expansão urbana acelerada, o que não impede que tais efeitos venham surgir no futuro;

- O avanço das áreas de VSA moderadamente alta poderá elevar o grau de degradação pelo aparecimento de novas áreas degradadas e pela expansão das já existentes, o que poderá causar impactos negativos à população;

- Partindo-se da metodologia adotada, outras variáveis podem ser incluídas em análises futuras a fim de aprofundar os resultados obtidos e propor cenários de manejo e conservação para a bacia do rio Japaratuba.

\section{REFERÊNCIAS BIBLIOGRÁFICAS}

AMARAL, S.; GAVLAK, A.A.; ESCADA, M.I.S.; MONTEIRO, A.M.V. Using remote sensing and census tract data to improve representation of population spatial distribution: case studies in the Brazilian Amazon. Population and Environment, v.34, p.142-170, 2012.

ARAGÃO, R.; ALMEIDA, J.A.P. Avaliação espaço temporal do uso do solo na área da bacia do Rio Japaratuba -Sergipe através de imagens LANDSAT. In: XIV Simpósio Brasileiro de Sensoriamento Remoto, 4, 2009, Natal. Anais... São José dos Campos, Instituto Nacional de Pesquisas Espaciais, 2009, p.1231-1238.

BARBOSA, C.C. et al. Operadores zonais em álgebras de mapas e sua aplicação no zoneamento ecológico econômico. In: SIMPÓSIO BRASILEIRO DE SENSORIAMENTO REMOTO, 8., 1996, Salvador. Anais... São Paulo: INPE, 1996. p.1-14. 1 CD-ROM.

BECKER, B.K.; EGLER, C.A.G. Detalhamento da metodologia para execução do zoneamento ecológico-econômico pelos estados da Amazônia Legal. Rio de Janeiro: SAE, 1997, 43 p.

FEITOSA, F.F.; CÂMARA, G.; MONTEIRO, A.M.V.; KOSCHITZKI, T.; SILVA, M.P.S. International Journal of Geographical Information Science, v.21, n.3, p.299-323, 2007.

FERREIRA, K.R.; CÂMARA, G.; MONTEIRO, A.M.V. An algebra for spatiotemporal data: from observations to events. Transactions in GIS, May, 2013.

FONTES, A.L. Caracterização Geoambiental da Bacia do Rio Japaratuba (SE). 1997, 298p. Tese (Doutorado em Geografia), Universidade Estadual Paulista Julio de Mesquita Filho, Rio Claro. 1997.

GONÇALVES, G.G.G.; DANIEL, O; COMUNELLO, E.; VITORINO, A.C.T.; ARAI, F.K. Determinação da fragilidade ambiental de bacias hidrográficas. Floresta. Curitiba, PR, v.41, n.4, p.797-808, out/dez 2011.

HALIM, R.; CLEMENTE, R.S.; ROUTRAY, J.K.; SHRESTH, R.P. Integration of biophysical 
and socio-economic factors to assess soil erosion hazard in the upper Kaligarang watershed, Indonesia. Land Degradation \& Development, Wiley InterScience, n.18, p.453-469, 2007.

HOLLY, M. Erosion and Environment (Sciences and Applications: Volume 9). Pergamon Press, Oxford, 236p., 1980.

HORNSBY, K.S.; COLE, S. Modeling moving geospatial objects from an ever-based perspective. Transactions in GIS, n.11, p.555-573, 2007.

INSTITUTO BRASILEIRO DE GEOGRAFIA E ESTATÍSTICA. Resultados do Censo Demográfico 2010. 2011. Disponível em: $<$ http:// www.censo2010.ibge.gov.br/resultados_ do censo2010.php>. Acesso em Jun. 2012.

JACOB, A.A.E.; YOUNG, A.F. O uso de métodos de interpolação espacial de dados nas análises sociodemográficas. In: XV Encontro Nacional de Estudos Populacionais, 11, 2006, Caxambu.
Anais... Belo Horizonte, Associação Brasileira de Estudos Populacionais, 2006, 22p.

MENEGOL, L.R. Paralelização de método de interpolação de dados: krigagem. 2004, 77p. Monografia (Curso de Ciência da Computação), Universidade de Passo Fundo, Passo Fundo. 2004. MOSER, C. The asset vulnerability framework: reassessing urban poverty reduction strategies. World Development, New York, v.26, n.1, 1998.

REARDON, S. and O'SULLIVAN, D. Measures of spatial segregation. Sociological Methodology, v.34, p.121-162, 2004.

SECRETARIA DE ESTADO DO MEIO AMBIENTE E DOS RECURSOS HÍDRICOS. Atlas Digital de Recursos Hídricos do Estado de Sergipe. Aracaju, SEMARH, 2011. 1 DVD-ROM.

WONG, D.W.S. Formulating a general spatial segregation measure. The Professional Geographer, v.57, p.285-294, 2005. 